\title{
A Rare Occurrence of Adrenal Leiomyosarcoma
}

Sharma A', Elisa Lewington-Gower ${ }^{1}$, Fausto Palazzo ${ }^{2}$, Ana Pokrajac ${ }^{1}$

${ }^{1}$ Department of Endocrinology, Watford General Hospital, West Hertfordshire Hospitals NHS Trust,

${ }^{2}$ Department of Endocrine Surgery, Hammersmith Hospital

\section{Clinical case}

-A 61-year-old Caucasian female was being investigated under the gastroenterology team for chronic abdominal pain. She reported pain and nausea triggered by sweets.

-CT scan of the abdomen and pelvis did not show any pathology other than an incidental $2.3 \mathrm{~cm}$ right adrenal nodule. She was then referred to our endocrine team.

-She underwent CT and MRI of the adrenals to further characterise the lesion. These were reported as 'indeterminate' but likely a benign adrenal incidentaloma.

- Further testing confirmed a non-secretory tumour (24 hour urine metanephrines [3 samples]: negative; normal aldosterone/renin ratio, ODST: $71 \mathrm{nmol} / \mathrm{L}$, normal LDDST)

\section{Follow-up}

- A follow up CT adrenal scan at 6 months showed an increase in the size of the adrenal nodule to $3 \mathrm{~cm}$.

- It was of heterogeneous density with delayed washout of contrast with a low-enhancing centre.

- The increase in size of the tumour within 6 months and the characteristics seen on repeat CT adrenal scan were suspicious of malignancy and she was referred urgently to the tertiary centre for a surgical opinion.

\section{Surgical findings}

- As the pre-operative investigations suggested an adrenal tumour, she was booked for a retroperitoneoscopic/laparoscopic adrenalectomy.

right

However at surgery the tumour was even larger and found to be invading the IVC and clearly malignant.

- A planned laparoscopic procedure was changed to an open procedure and a grade 2 right peri-adrenal leiomyosarcoma was resected with resection of the lateral wall of the IVC.

- Interestingly, the attached adrenal gland was normal.

- Her sweet intolerance resolved after surgery.

- She is currently followed up in a dedicated leiomyosarcoma centre.

- Macroscopic description: Tumour measured $64 \mathrm{~mm}$ by $40 \mathrm{~mm}$ by $38 \mathrm{~mm}$. Ki67 proliferation fraction: up to 90\%. Immunohistochemistry: tumour cells positive for SMA, desmin, MNF116 and h-caldesmon. Negative for S100p, Melan A, Inhibin, Calretinin and synaptophysin.

Zhou Y et al. Primary adrenal leiomyosarcoma: a case report and review of literature. International Journal of Clinical and Experimental Pathology. 2015;8(4):4258-4263.
Dutta P et al. Non-islet cell tumor-induced hypoglycemia: a report of five cases and brief review of the literature. Endocrinology, Diabetes \& Metabolism Case Reports. 2013-2013:130046.

Figure2: CT adrenal with contrast July 2015:

$3 \mathrm{~cm}$ by $3 \mathrm{~cm}$ right adrenal nodule.

Pre-contrast: $37 \mathrm{HU}$; early contrast 50HU; delayed contrast $60 \mathrm{HU}$

\section{Figure1: CT adrenal with} contrast Feb 2015: $2.3 \mathrm{~cm}$ right adrenal nodule. Pre-contrast: 48HU; early contrast 48-53HU; delayed contrast $81 \mathrm{HU}$

\section{Results}

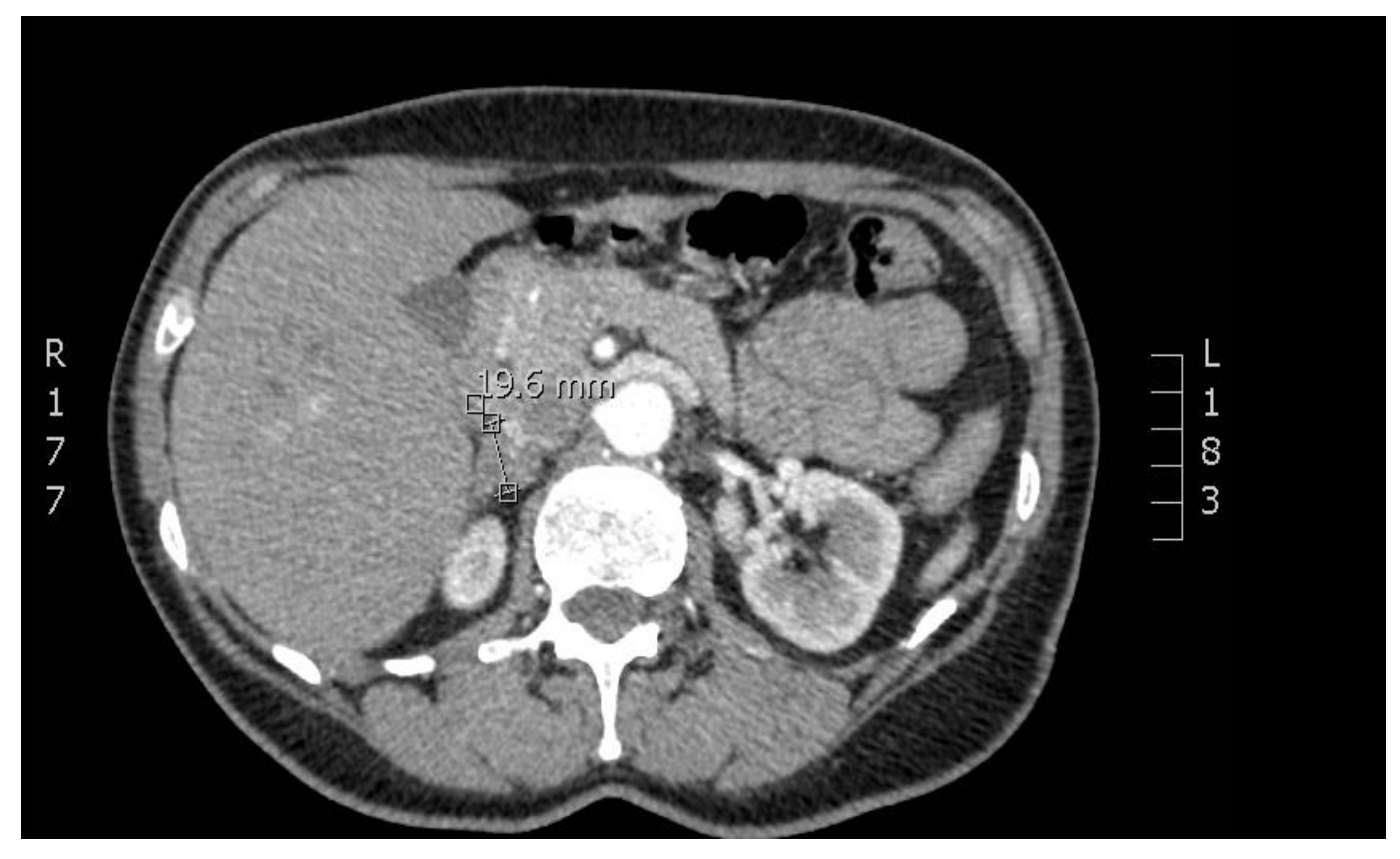

Figure 3: Histology

This $H$ \& $E$ image is of the

tumour at a higher $x 10$ magnification showing spindle cells exhibiting features of malignancy including mitotic activity and nuclear pleomorphism

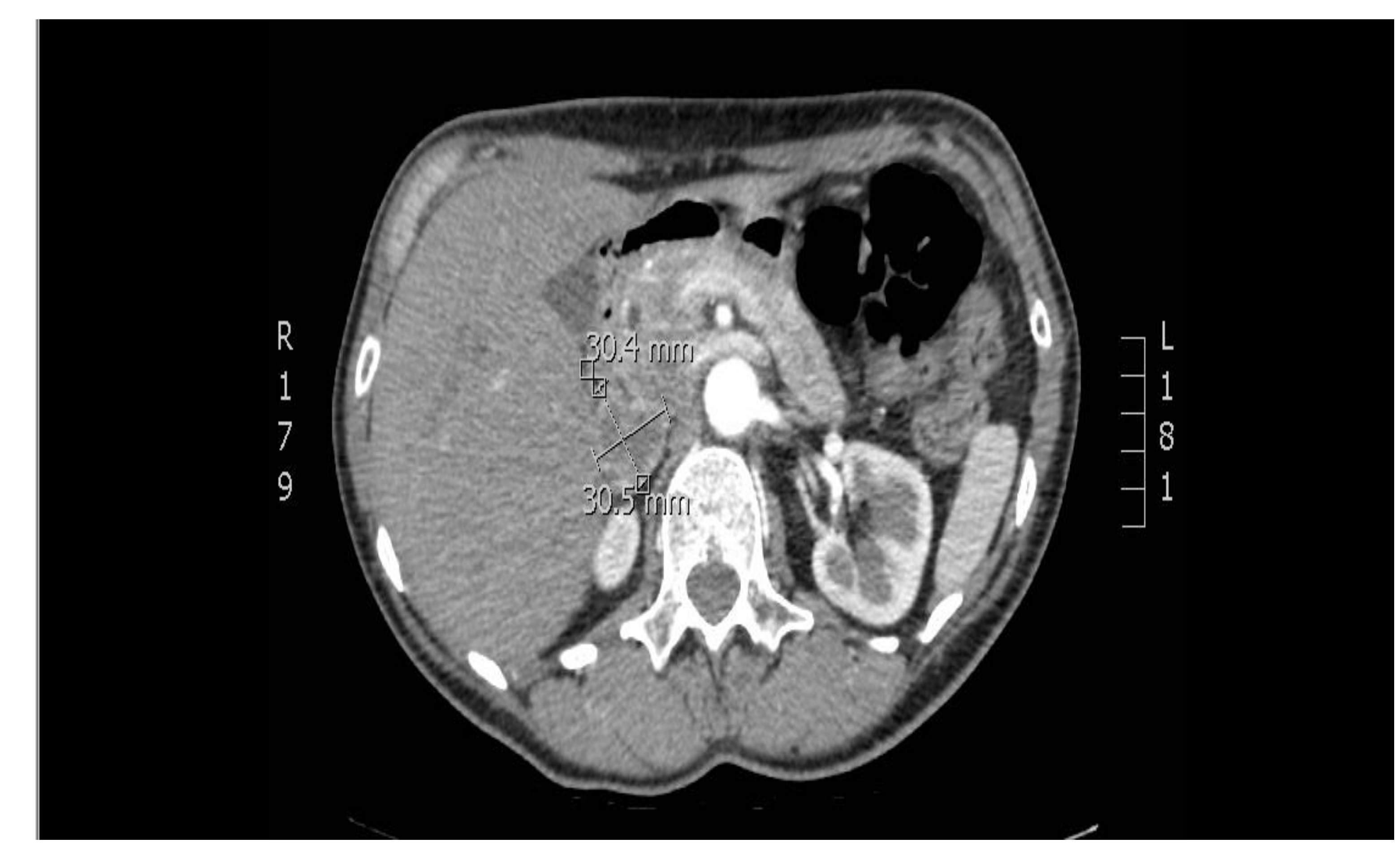

\section{CONCLUSION}

* Mesenchymal tumours like leiomyosarcoma are associated with non-islet cell tumour-induced hypoglycaemia caused by the unregulated production of IGF-II and extensive glucose metabolism.

* This case highlights the importance of appropriate radiological assessment in adrenal incidentalomas by experienced adrenal radiologists.

* Monitoring of patients with suspicious looking lesions even if not meeting the initial criteria for surgery should be rigorous and discussed in a dedicated multidisciplinary team. 